\title{
The Transformation of the Online Teaching Role of University Teachers Under the Embodied Cognition Perspective
}

\author{
Lei Yue ${ }^{1}$
}

\author{
${ }^{1}$ Harbin Normal University, \\ Institute of Education science \\ Harbin, China \\ 2196336232@qq.com
}

\begin{abstract}
With the gradual intervention of the online teaching process of artificial intelligence, the virtual three-dimensional space and the traditional physical space gradually integrate and promote the reshaping of the teaching environment. The teaching needs of the era based on educational big data have put forward new requirements for the classroom role of university teachers. Firstly, from the perspective of teaching environment, teaching subject, cognitive science, then, the teaching enlightenment is summarized to build appropriate learning environment, appropriate learning status, multiple learning experience, technical design, content guidance, activity organization and habit guidance, including environment, experience, subject and activity.
\end{abstract}

Keywords : Physical cognition, role of teacher, online teaching.

\section{INTRODUCTION}

Since the 19th century, Western philosophical theories have poured in. Among them, the school of technical phenomenology represented by Tang Eman and Heidegger took the lead in producing perspective-related ideas on technology, and thus gave birth to the second generation of cognitive science. In the context of the first generation of cognitive science, the emerging cognitive theory of "embodied cognition" has emerged as the representative theory of the second generation of cognitive science. ${ }^{[1]}$

With the continuous exploration of educators and the gradual maturity of the ideological system, embodied cognition was introduced into the field of psychology, and it began to enter the field of pedagogy after receiving further confirmation of cognitive neuroscience and experimental psychology. In the new field of education with the support of artificial intelligence technology, how to redefine the existence value and role positioning of teachers, especially builders and managers of Higher Education -- University teachers, has become an urgent key problem to be solved to promote the development of future education.Nowadays, the constructivist theory of constructivism lacks the basis to respond to the role task of online teaching teacher that is highly compound and interactive, and a theory that emphasizes the integration of human brain itself and technical environment is needed to guide the reshaping of teacher role in the online teaching environment.

As a derivative theory of technical phenomenology, the self-equipped cognition theory emphasizes the feeling and exploration of the human body itself in the learning process, attaches importance to the interaction between people and the environment in the learning environment, and provides new enlightenment for the role transformation of university teachers in today's online teaching ${ }^{[2]}$. In online teaching research, the exploration of teacher role has gradually attracted the attention of researchers, and is believed to be closely related to the construction of learning effect.Therefore, the online role transformation of university teachers under the guidance of the physical cognitive perspective gradually highlights its value. 


\section{RELEVANT TEACHING THEORY AND PRACTICAL ENLIGHTENMENT OF PHYSICAL COGNITION}

The embodied cognitive theory first originated from Husserl's debate with Descartes over the dualism of the mind. In his reflection on Cartetes's "physical and mental dualism", Husserl attracted philosophical attention to body perception, life form and tactile experience. Based on this, Heidegger took existence itself as the starting point, thought phenomenologically, and believed that human understanding of the world is realized through the interaction of our body with other objects in the world ${ }^{[3]}$ Since then, another philosopher Meloponti has expounded the neutral "monistic" view by discussing the identity of perception (phenomenon) in the world, believing that the mind and body are inseparable from the world they live in, and emphasizing the dynamic unity of cognition, body and environment ${ }^{[4]}$ 。

On this basis, Lekoff and Johnson distinguish the paradigm of cognitive science and define their development stage, namely "detached cognitive science" and "embodied cognitive science", respectively, formally putting forward the concept of physical cognition. ${ }^{[5]}$ With the further development of neuroscience, the discovery of mirror neurons also provides a theoretical basis for embodied cognition. The concept of embodied learning developed thus treats people as a whole that can perceive, perceive, act and think, and is able to understand ourselves and the world around us, rather than an abstract object or tool. Thereafter, Wilson summarizes six points of embodied cognitive field: (1) cognition is context-based; (2) cognition is time stressful; (3) should develop environment to reduce cognitive load; (4) environment is part of cognitive system, mind alone does not constitute an important analysis unit; (5) cognition is guiding behavior; (6) offline cognition is body-based ${ }^{[6]}$.

\subsection{Correlation theory}

After the Wlison, there are also scientists and educators who continue to explore the embodied cognitive theory, and constantly explore their feasibility in various fields. Therefore, embodied cognitive theory has gradually extended from the field of psychology to pedagogy, linguistics and other fields, and has been widely recognized in various scientific fields. To sum up, various representative theories of physical cognition come from four aspects: teaching environment, teaching subject, teaching experience and teaching activities. Therefore, the author classifies the relevant theories and discussions into the following four aspects:

\subsubsection{Teaching environment}

Physical cognitive theory believes that the teaching environment plays an important role in promoting learners' learning perception. For example, Merponti pointed out in his work perceptual Phenomenology that the mind, body and environment are unified to complete the construction of the learner cognitive process; the learning environment should pay attention to the learner itself in the environment, and constantly produce generative changes, that is, identity and subjectivity ${ }^{[7]}$.In addition, the learning environment should not only include the organization of teaching activities, but also pay attention to physical and emotional participation, which is especially important for the learning of knowledge about concepts and principles in the field of basic education.It can be seen that the embodied environment is the medium to promote the reorganization of learners' experience, a tool to organize the learning process with the body as the link, and a cognitive platform for the interaction with other teaching factors.

\subsubsection{Teaching subjects}

As early as the 1940s, Husserl had proposed a prior ontology, emphasizing tactile forms and life experience in cognitive processes; later Heidegger proposed "man" as the starting point of all research, emphasizing the importance of learners in the teaching process ${ }^{[8]}$ 。 "Learning subjectivity" has always been the key content of the field of embodied cognition. Today, Robb Lindgrena et al. are still committed to designing teaching systems with learners and developing learners' independent exploration ability in the learning process. Therefore, it is clearly seen that taking learners as the teaching subject is an indisputable consensus in the cognitive field, and embodied learning is the learning paradigm of learners as the subject. Therefore, in the process of embodied teaching, attention should be paid to the play of learning subjectivity.

\subsubsection{Teaching activities}

Moderate teaching activities are an important medium to make learners participate in teaching activities physically and mentally, and also the maintenance factor of physical learning subjectivity. In Meloponti's discussion, appropriate perceptual activity is the link for the reorganization of the perceptual fields; moreover, studies on mirror neurons show that moderate teaching activity provides learners with the opportunity to participate in learning and guides them to form complex cognitive schema ${ }^{[9]}$.The design of moderate teaching activities with learners as the main body, acting as a learning medium in the organizational activities of embodied teaching, promotes the development of learners' cognition. Therefore, moderate teaching 
activities should be paid attention to in the embodied teaching.

\subsubsection{Teaching experience}

Physical cognitive theory believes that the diversity of teaching experience depends on the integration of teaching channels, and plays a crucial role in the learning effect of learners.More than 90 years ago, educator Montessori advocated for multi-channel teaching and learning, arguing that learning should include vision, hearing, touch, and movement, not just traditional speaking and listening.In modern research, Mayer, a cognitive scientist, put forward multimedia cognitive theory, believing that teaching through a variety of perceptual teaching media is conducive to improving the absorption rate of learners and deepening learning memory and experience ${ }^{[10]}$.Under multiple sensory stimulation, learners' sensory channels expand, and then the absorption of knowledge is enhanced, allowing for faster entry into the heart current state. Many theories confirm that,Multi-channel integrated teaching and learning, especially sports assistance, can help learners improve learning experience and thus learning effect.

\subsection{Summary of teaching enlightenment}

According to the above embodied cognitive teaching theory induction, the author summarizes the teaching enlightenment as follows:

\subsubsection{Build a physical learning environment}

As mentioned above, the mind, the body and the environment are an organic and unified whole, and the complete embodied cognition and learning process cannot be separated from the shaping of the embodied learning environment. Learning environment is the platform for learning, the background of all teaching, and provides a foundation for the development of teaching.

The interaction between learners' senses and the environment is the cornerstone of the formation of advanced cognitive development and relies on the construction of an embodied learning environment. Physical learning environment refers to a learning environment that can help learners to conduct experience reorganization and schema reconstruction through their own perception or experience, and constantly undergo generative change according to their cognition of participation.It values the subjectivity and environmental fitness of the learners, and reflects the emphasis on the flesh in the Husserl prior ontology. At the same time, it also expands the perceptual range and immersive experience of learners, which is crucial to achieve embodied teaching and improve teaching efficiency.

\subsubsection{Emphasizes student principal status}

From the perspective of the relationship between knowledge and cognitive objects and subjects, the core idea of embodied cognitive theory construction is "body-related", that is, it emphasizes the learners' perception of subjects in the learning process. The theme perception of learners has always been the focus of the embodied school of cognitive theory.

Promoting the organic link between learners and the teaching environment and teaching content is the key to physical cognition. In physical learning, knowledge generation and schema transformation need the support of learners' body structure, external perception experience, and neural structure changes; at the same time, the knowledge difference in the learning process is also reflected by the connection of knowledge background and body perception. Moreover, the nature of embodied cognition emphasizes the learner's own experience as the construction basis of the learning process. Therefore, the learner's own experience is a necessary step to build on in an embodied environment, is the experience basis for cognitive development, and the role of its main status is self-evident.

\subsubsection{Carries out appropriate learning activities}

From the perspective of the participation relationship of physical and mental consistency, the unity of mind and body is an important core of the construction of physical learning significance. The principle of physical and mental consistency of embodied teaching requires educators to try to make learners physically and mentally participate in teaching activities in the teaching process. As a necessary procedure for the teaching process, learning activities are a feasible way to reflect the role of teachers and enhance the enthusiasm of learners.

The development of suitable learning activities is the embodiment of effective teaching intervention in embodied teaching. It is the embodiment of the course knowledge to vividly display the theme and content of teaching activities and guide the learners to participate in the generative learning process. In the development of learning activities, learners conduct research learning exploration based on the effective information provided by teachers, linking external perception and memory mechanisms organically, and ultimately promoting the transformation of cognitive schema and behavioral mode. Therefore, the development of moderate teaching activities can help learners to better complete physical learning and improve the effectiveness of knowledge construction. 


\subsubsection{Values multiple learning experiences}

From the definition of embodied learning itself, learning experience is the direct source of learners' knowledge. Diversified learning experience content is the source of cognitive diversity and difference among learners.

In Meloponti's view, the process of learning is the unity of multiple functions. The unification of multifunctional energy puts forward the diversity and multisource of learning, which requires a variety of learning experience under the construction of the first three, learning environment, learning subjects and learning activities. A variety of learning experiences can not only meet the richness of multi-functional learning, but also make up for the individual differences in learning. It is an important way to promote individual cognition and improve learning efficiency, and an effective targeted strategy for individual perceptual diversity in embodied learning.

\section{TRANSFORMATION OF THE TEACHING ROLE OF UNIVERSITY TEACHERS IN TEACHING FROM A COGNITIVE PERSPECTIVE}

Under the background of the rapid development of the Internet, artificial intelligence gradually intervenes in the process of online teaching, and the complicated learning needs and network tools jointly give rise to the changes of students' teaching needs and promote the rise of online teaching. Especially during the COVID-19 period, online teaching was considered as the ideal teaching way for most schools, and it has ushered in once-in-a-lifetime development opportunities. However, in the teaching activities launched under the background of the epidemic, many teachers are faced with the role transformation due to environmental transformation and network Settings, etc ${ }^{[11]}$. The resulting series of problems gave rise to research on the role of university teachers in online teaching.

\subsection{Requirements of college teaching from the offline teaching perspective}

Compared with offline teaching, online teaching has changed due to its environmental setting and platform operation, and the teaching requirements for university teachers have also changed ${ }^{[12]}$. Therefore, the teaching requirements of teachers in teaching have also changed accordingly, in order to play the role of teachers as much as possible in the classroom on the premise of achieving the teaching effect.Based on the above review structure, the author summarizes the teaching requirements of online universities as follows:

\subsubsection{Construct a generous teaching environment}

Compared with traditional offline professors, with the participation of online network technology, the closure of the teaching environment is broken, and the communication nature and information diversity are prominent. Based on the construction of an Internet learning platform, students can learn classroom knowledge through various channels, and the convenience of communication with other similar learners has been greatly improved.

Therefore, as a university teacher, we should not only calmly deal with the "release" in online teaching, that is, exercise the divergence of students 'thinking through open communication and sharing; but also grasp the "collection", that is, through the construction of a certain classroom environment, grasp the boundary of classroom teaching, exercise the convergence of students' thinking. ${ }^{[13]}$ This is not only the literacy requirement of the instructor, but also the only way for the network teaching.

\subsubsection{Pay attention to the students' main position of teaching}

In the process of online teaching, it is the choice of many university teachers to teach based on the existing teaching platform. In the construction of the network teaching platform, many online teaching platforms adopt adaptive systems, that is, student-centered teaching activities and teaching curriculum setting. At the same time, because the amount of information available to students mentioned above increases greatly, the process and results of students to independently obtain information are particularly important.

Therefore, as the trainer and guide of college students 'research ability and learning ability, university teachers should pay attention to the cultivation of college students' subjectivity in the teaching process. In other words, university teachers should pay attention to changing teaching thinking and carry out teaching design and organization with students as the main body.

\subsubsection{Relies on environmental science teaching activities}

In the online teaching environment, based on the above two points, the teaching activities require teachers to make greater adjustments. Under the premise of leaving the on-site communication and organization of face-to-face teaching, online teaching activities rely more on the technical organization in the environment, so as to improve students' attention and the degree of physical learning. 
Therefore, as a university teacher, we should make full use of the technology of the teaching environment and the subjectivity of teaching activities. It is embodied as: the combination of teaching activities and teaching content as the link between students' learning, to promote students to have research and generative learning, so as to complete the requirements of online teaching.

\subsubsection{Guides good learning habits}

As described in the above three points in common, the teaching development in the network environment needs to be based on the students' autonomy and subjectivity. From this point, teachers need to guide students to form good online learning habits, in order to maximize the role of online teaching.

To sum up, as a university teacher, the online teaching learning activities should realize the role transformation from four aspects: building the teaching environment, adjusting the main position, building the teaching activities, and guiding the habit formation, so as to achieve the teaching effect. Therefore, the transformation will be summarized as follows:

\subsection{Transition from skilled technology user to technical environment designer}

In the traditional structuralist teaching design theory, teachers have full control of the students' learning process and can carry out fine control, completing the teaching process step by step as the engineer completes the project ${ }^{[14]}$; The ability to use the Internet only exists as a vassal of the teaching ability. However, when the Internet penetrates into the university classroom, the diversification of cultural, language and technological means makes the knowledge that students accept and pass on daily appear in various forms, and the skilled use of teaching techniques has become the foundation and cannot meet the learning needs of learners.

As mentioned above, the occurrence of effective learning is based on the natural environment where the cognitive subject is fully integrated with the physiological resources within the body.Open online teaching and diverse teaching conditions and environment provide a foundation for teachers to design a suitable learning environment, which can help students to obtain learning experience and feelings through real experience in the virtual three-dimensional space. In addition, in online teaching, students' autonomy in gaining access to knowledge has received broader technical support due to the diversity of their knowledge sources and the openness of the knowledge system. Therefore, it is an effective way to design an excellent university online learning environment by using the multidimensional advantages of online teaching to improve students' learning efficiency. To sum up, the role of a teacher from a single skilled manipulator to the designer of the teaching technology environment is the general trend for students to promote effective learning from the cognitive perspective.

\subsubsection{Changes from the leader of the teaching process to the mentor of the teaching content}

In the learning view of physical cognition, the knowledge received through their own feelings, at the same time, our physical cognitive scholar Ye Haosheng believes that each learner's learning cognition is the unity of body, brain and environment ${ }^{[15]}$, Therefore, students will have different learning needs based on their own experience and body perception.

Moreover, the process of teacher-dominant online teaching is weakened due to its own characteristics. In the traditional constructivist teaching design, teachers, as "engineers", accurately control students 'learning process to meet the characteristics of systematic learning. In the process of online teaching, the diversified characteristics of the Internet itself meet students' needs to obtain multi-perspective knowledge and reduce the single source of classroom knowledge. College students are the main body of the higher education system, and their own development characteristics require that they should not only master the knowledge of this stage, but also have the research ability to match their own knowledge ${ }^{[16]}$.

To sum up, from the perspective of physical cognition, personalized and differential attention is the focus of online higher education, which is bound to put forward new requirements for the role of university teachers, and becoming a teaching guidance rather than a dominant person is an important choice to cultivate students' comprehensive grasp of knowledge and meet the needs of education.

\subsubsection{Change from the controller of the overall teaching process to the organizer of multiple teaching activities}

The classroom teaching under the guidance of the embodied cognitive concept advocates that students "mobilize the participation of body, mind and consciousness through personal experience, and obtain the all-round development of cognition, emotion and skills" in learning activities. In the process of the activity, students take the initiative to think about themselves, explore together and learn under the guidance of teachers.

Compared with the traditional teaching design, teachers have to master the whole teaching process accurately. In addition to the above advantages of multichannel learning from the physical cognitive perspective, the personalized learning methods with students as the 
main body in online teaching also need various teaching activities to meet students' individual learning needs. The overall open style of online teaching and the convenience of acquiring knowledge also provide conditions for the design of various teaching activities. Furthermore, presenting the teaching process in colorful teaching activities rather than requiring teachers to fully manipulate the entire teaching process reduces the teacher burden from the perspective of the energy allocation.

\subsubsection{Change from the layout of the teaching task to the guide of learning habits}

In the guiding concept of physical cognition, learners, as the main body of the learning process, their ability to actively explore and learn plays a crucial role in their learning effect. For example, Merponti pointed out in his theory that human learning and cognitive processes are the unity of brain activity, body perception and environmental factors ${ }^{[17]}$. Therefore, the role of learning habits in assisting learners to complete learning is obvious.

Compared with offline teaching activities, online teaching activities are more open and have more uncontrollable factors. Therefore, different from offline teachers who can grasp the overall situation and realtime supervision, online teaching needs teachers to guide the change of students' learning behavior, so as to use the network for learning to the greatest extent on the basis of giving full play to their autonomy. To sum up, on the basis of the above role change of teachers, teachers should also be the guide of students' habits. In online learning activities, teachers can guide the change of student habits in many ways. First of all, teachers should first do a good job of behavior, to establish a good model of good learning. At the same time, good learners are selected as representatives to play a leading role; Secondly, it is that the teacher appropriately restrict the use of learners' Internet network tools during class, or formulate flexible rules and regulations to appropriately restrict the use of the Internet during class. In addition, a good online learning environment is also the key to develop learning habits. Teachers should choose the teaching platform reasonably according to the situation, so as to adapt to the needs of teaching and learning, so that learners can focus on the classroom during class time, and develop the habit of concentrating on class.

\section{CONCLUSION}

From the perspective of physical cognition, this paper first expounds the development process and history of embodied cognition theory, and summarizes the enlightenment of offline teaching from the physical cognitive perspective. On this basis, this paper compares the differences between online and offline teaching, and expounds the transformation of online teaching role under the guidance of university teachers from four perspectives.

The theory of embodied cognition has been continuously developed and improved since its birth in the 1950s, but it always adheres to the study of interactive experience research between people and the environment and cognition, which provides a reference for online teaching. The study of the integration of physical experience and auditory, visual, language in this theory inspires educators to think and discuss how to integrate information education that supports multichannel integration into embodied teaching.

In the teaching process, educational technology itself also guides guidance to educators. Teachers use the teaching technology in the classroom, and the teaching technology also imperceptibly affects the subject cognition of the teacher role, and promotes the transformation of the teacher role ${ }^{[18]}$. From another point of view, this has triggered a thinking on the application of teaching technology in offline teaching. With cognition as the core of the future education mode is under the guidance of the traditional teaching mode on the body and the environment, gradually to the way of cognitive, body, environment combination, but also reshape the connotation of the teacher role in the process of teaching, for the role of provide a new reference for classroom teachers reference significance.

\section{REFERENCES}

[1] Li Zhihe, Li Pengyu, Zhou Nana, Liu Zhixiu. Cognitive Learning Environment Design: Features, Features, Application and Development Trends [J]. Distance Education Journal, 2018,36 (05): 81-90.

[2] Chen Xingye, Li Man.. Teaching Model Design for Information Technology with Physical Knowledge [J]. Open Education Research, 2020,26 (02): 111 119.

[3] Fan Wenxiang, Zhao Libin.. Knowledge, learning and teaching views [J]. Electrical Education Research, 2020,41 (07): 21-27+34.

[4] Yang Yujun., English Teacher Training Research Based on a Physical Cognitive View [J]. Foreign Language, 2020,36 (03): 138-144.

[5]. Alstitution and transcendence of Virtual Teaching AIDS: [J]. Electrical Education Research, 2020,41 (12): 50-58.

[6] Zhang Lu, Shang Junjie. Gaming Learning Theory Research Based on the Learning Experience perspective $[\mathrm{J}]$. Electrical Education Research, 2018,39 (06): 11-20 + 26 . 
[7]. The path of traditional culture into ideological and political courses [J]. Middle School, 2020 (38): 5154.

[8] Chen Fengyan, Zhan Licai, Peng Yuji, Meng Shimin-Based Deep Learning Research — Take the "Morality and Society" course [J]. Modern Educational Technology, 2019,29 (10): 121-126.

[9] Zhou Huixin. Physical Recognition of Modern Physical Teaching in the Vision of Body Philosophy [J]. Chinese Education, 2017 (08): 4145.

[10] Wang Yixiao. Theory of embodied cognition: Contextual Interaction [J]. Electrical Education Research, 2018,39 (07): 20-26.

[11] Shen Zhen. Online Teaching Strategies in Ideological and Political Theory Courses in Colleges and Universities under Epidemic Prevention and Control $[\mathrm{J}]$. Ideological and Theory Education Guide, 2020 (03): 15-19.

[12] Yang Lu, Jiang Ke. Current situation, problems and reflection of online teaching in higher vocational colleges during the Epidemic Prevention and Control period [J]. Education and Vocational, 2020 (13): 99-103.

[13] Zheng Xudong, Wang Meiqian, Rao Jingyang. On Learning and Design: Based on cognition [J]. Electrical Education Research, 2019,40 (01): 25-32.
[14] Ye. Body and Learning: Physical Recognition and Its Challenges to Traditional Education [J]. Education Research, 2015,36 (04): 104-114.

[15] Li Zhihe, Li Sizhe, Wang Yuanchen, Zhang Chunyu. Design and Verification of the Deep Learning Assessment Scale for College Students [J]. Electrical Education Research, 2020,41 (12): 92-98.

[16] Xia Xiaoyan, Shi Hongmin, Guo Yaling, Xie Chengkai, Guo Helu. VR Curriculum Teaching Principles and Top Design of Foreign Language Teaching $[\mathrm{J}]$. Foreign Language Electronic Teaching, 2021 (02): 78-84 + 12 .

[17], George Bailey. Educational Drama in Cognitive Theory: Physical Phenological View [J]. Theory, 2021 (06): 154-160.

[18] Li Zhihe, Li Sizhe, Wang Yuanchen, Zhang Chunyu. Design and Verification of the Deep Learning Assessment Scale for College Students with physical cognition [J]. Electrical Education Research, 2020,41 (12): 92-98.

[19], Forrest. Primary and Secondary Education in Major Epidemics: Based on empirical analysis [J]. Education and Economics, 2020,36 (02): 3-9 + 28. 